\title{
VARIACIÓN EN EL CRECIMIENTO TEMPRANO EN ALTURA DE ORIGENES MARGINALES ESTEPARIOS DE CIPRÉS DE LA CORDILLERA
}

\author{
Aparicio Alejandro ${ }^{1}$, Pastorino Mario ${ }^{12}$ y Gallo Leonardo ${ }^{1}$
}

\section{RESUMEN}

El ciprés de la cordillera, Austrocedrus chilensis (D. Don) Pic. Ser. et Bizzarri, es una conifera nativa de gran importancia forestal en la región patagónica. Con el objetivo de analizar variación temprana en caracteres cuantitativos se estableció un ensayo de ambiente común en invernáculo, con regulación automatizada del ambiente y un régimen de ferti-irrigación por micro-aspersión ajustado para la especie, durante dos temporadas de crecimiento. Se utilizaron 158 progenies de 10 poblaciones, elegidas al azar, representando a toda la distribución latitudinal del tipo forestal marginal estepario, que ya se ha destacado en estudios previos por su importancia para el uso y la conservación de los recursos genéticos de la especie. Veintiuna plantas por cada tratamiento se distribuyeron en un diseño aleatorizado de bloques completos (3 bloques). Se midió la altura desde la inserción de los cotiledones hasta la yema terminal del eje principal en 9 ocasiones, comprendiendo la totalidad del segundo periodo de crecimiento. Se analizaron los componentes de la varianza para las alturas inicial y final, con un modelo anidado de efectos aleatorios. Se ajustaron modelos de crecimiento, y se efectuaron comparaciones entre progenies utilizando medidas de información (Criterio de Información de Akaike).

La altura al final de la segunda temporada de crecimiento tuvo un promedio de $216 \pm$ $65 \mathrm{~mm}$, con un minimo y un máximo absolutos de 65 y $512 \mathrm{~mm}$ respectivamente (la longitud promedio del hipocótilo fue de 19,3 \pm 4,9 mm). Las alturas iniciales y finales del segundo periodo de crecimiento estuvieron altamente correlacionadas en el $55 \%$ de las progenies. Aproximadamente un $23 \%$ de la variación se debió al efecto de las progenies, mientras que sólo un $3 \%$ se debió al origen. El $74 \%$ restante se atribuyó principalmente a la variación entre individuos dentro de las familias. El crecimiento en altura se ajustó a modelos polinomiales de segundo grado, que a nivel de progenies tuvieron coeficientes de determinación de entre 0,51 y 0,92 . Las curvas de crecimiento difirieron en al menos un parámetro en el $75 \%$ de los contrastes entre progenies de igual origen. Las diferencias se encontraron mayormente en las ordenadas al origen (alturas iniciales del periodo analizado), pero tambièn en las pendientes (tasas de crecimiento) de las curvas.

Los resultados de esta evaluación temprana sugieren que, por criterios de productividad, para un plan de mejoramiento genético se deberia dar preponderancia a la selección de progenitores antes que de origenes. La utilización de modelos de crecimiento que permiten

1-Instituto Nacional de Tecnologia Agropecuaria (INTA), CC 277 (8400) Bariloche, Argentina, aaparicio@bariloche.inta. gov.ar

2-Consejo Nacional de Investigaciones Cientificas y Técnicas (CONICET). Argentina 
cuantificar la tasa de crecimiento (pendiente de la curva) podria aportar mayor información y ser más eficiente para la selección temprana de progenies, que la evaluación basada sólo en la altura final, ya que esta variable podria estar influida por efectos maternos propios del primer año.

Palabras clave: Austrocedrus chilensis, ciprès de la cordillera, progenies, crecimiento inicial.

\section{SUMMARY}

Patagonian Cypress, Austrocedrus chilensis (D. Don) Pic. Ser. et Bizzarri, is a regionally very important conifer native to Patagonia. Its marginal forest type from the steppe was found to be essential for conservation and breeding objectives of the species. With the purpose of analysing early variation in quantitative traits, a common garden trial was established in a greenhouse, under automatically regulated conditions and a ferti-irrigation regime adjusted for the species, for two growing seasons. In this study, 158 progenies corresponding to 10 randomly selected marginal populations encompassing its whole latitudinal distribution in Argentina, were assayed. Twenty-one seedlings per treatment were distributed in a randomised complete block design ( 3 blocks). Height from the cotyledons insertion till the terminal bud of the main axis was measured 9 times along the whole second period of growth activity. Variance components were analysed for initial and final heights, using a nested randomised effects model. Growth models were adjusted, and comparisons between progenies were made using information measures (Akaike's Information Criterion).

Mean height at the end of the second growing season was $216 \pm 65 \mathrm{~mm}$, with absolute minimum and maximum values of 65 y $512 \mathrm{~mm}$, respectively (mean hypocotyl length was 19.3 $\pm 4.9 \mathrm{~mm}$ ). Initial and final heights of the second growing season were highly correlated in 55 $\%$ of the progenies. Out of the total variation, $23 \%$ was caused by the effect of the progenies, while only $3 \%$ was due to origins. The remaining $74 \%$ was attributed mainly to variation among seedlings within the progenies. Height growth fitted second degree polynomial models, with coefficients of determination between 0.51 and 0.92 at the progeny level. Growth curves differed in at least one parameter in $75 \%$ of the contrasts between progenies of the same origin. Differences were found mainly in the origin to the ordinates (i.e. initial height for the analysed period), but also in the slopes (i.e. growth rate) of the curves.

The results of this early evaluation suggest that, considering productivity criteria, progenitors more than origins should chiefly be selected in a breeding program for the species. Besides, the use of growth models which allow quantify the growth rate based on the slope of the curves, seems to be more informative and efficient for the early selection of progenies than evaluations based on final height only, since this variable could be influenced by maternal effects typical of the first growing season.

Key words: Austrocedrus chilensis, ciprés de la cordillera, progenies, early growth. 


\section{INTRODUCCIÓN}

El ciprés de la cordillera Austrocedrus chilensis (D. Don) Pic. Ser. et Bizzarri es la conifera nativa de mayor importancia de la Patagonia argentina, ocupando sus bosques en la actualidad una superficie aproximada de 141.000 hectáreas (Bran et al., 2002). La madera de ciprés, proveniente exclusivamente de los bosques nativos, constituye un producto forestal muy valorado a nivel regional, con una demanda creciente y con el precio más alto entre las maderas de coniferas comercializadas en la región. Existe un creciente interés en el sector forestal regional, en la producción comercial con especies nativas, entre ellas el ciprés, motivo por el cual resulta necesaria la domesticación de dichas especies para lograr su uso en plantaciones comerciales. Si bien el ciprés es una especie de crecimiento lento en comparación con las coniferas exóticas cultivadas en la región, se estima que la potencialidad productiva con material mejorado seria buena, habiéndose reportado incrementos de entre 12,2 (Loguercio et al., 2005) y $16 \mathrm{~m}^{3} / \mathrm{ha} / \mathrm{año}$ (Veblen et al., 1995), sin mejoramiento genético ni manejo silvicola.

El ciprés ocurre en un amplio gradiente ambiental determinado principalmente por las precipitaciones, y es la especie forestal que más se adentra en la estepa patagónica, llegando a encontrarse pequeños bosquetes o árboles aislados hasta en sitios con $300 \mathrm{~mm}$ de pma (precipitación media anual). La adaptación natural del ciprés a ambientes xéricos extremos representa una ventaja para el uso de la especie en plantaciones para diversos usos, en áreas de ecotono entre el bosque y la estepa. El tipo forestal marginal estepario (pma de entre 300 y $490 \mathrm{~mm}$ ), fue descripto como el de mayor variación genética a través de estudios mediante marcadores genéticos (isoenzimas) (Pastorino, 2001; Gallo et al., 2004). Asimismo este tipo forestal ha mostrado buen comportamiento adaptativo fuera de su ambiente natural, en caracteres de germinación y crecimiento temprano (Leonardo Gallo, datos sin publicar). Por los motivos mencionados los bosquetes marginales esteparios tienen una gran relevancia para la conservación y el uso de los recursos genéticos del ciprés.

La utilización del ciprés con fines comerciales, requiere de la determinación de las mejores fuentes genéticas para maximizar los beneficios. La determinación de las mejores fuentes geográficas o áreas de origen, asi como la definición de las estrategias más adecuadas para la selección, se basan en el análisis de la variación para caracteres adaptativos de importancia económica.

Desde el punto de vista productivo, el crecimiento inicial en altura es uno de los caracteres de mayor importancia para el éxito de una producción forestal. Esta caracteristica determina los costos iniciales de producción de plantines en vivero, y de la fase de establecimiento de la plantación. Mayores tasas de crecimiento inicial incidirán no sólo en el incremento volumétrico alcanzable, sino en los costos de protección inicial de la plantación, debido a la disminución del tiempo de competencia con malezas ó con plantas nodriza, y del tiempo en que las plantas son susceptibles de daños por plagas como liebres y conejos. En sistemas productivos mixtos con ganado, la plantación de material con mayores tasas de crecimiento inicial disminuiria los tiempos de clausura de las àreas implantadas.

La selección por caracteres de crecimiento suele llevarse a cabo a través de análisis comparativos entre las diferentes entidades probadas en ensayos de ambiente común, utilizando 
análisis de la varianza y tests de comparaciones múltiples a posteriori (e.g. test de Tukey), o algún valor umbral a partir del cual seleccionar. Este enfoque permite identificar grupos de tratamientos cuyas medias para el carácter en cuestión no difieran a un determinado nivel de significancia. La variación en altura inicial, como ejemplo de variable cuantitativa de selección, puede ser evaluada con este enfoque utilizando una medición estática de dicha variable. Sin embargo, esta variable vista como un estado estático, podria estar influida por efectos ajenos a la genética o por efectos de muy corto plazo, tales como correlaciones entre el crecimiento inicial y el peso de la semilla, el año de cosecha, los tiempos de germinación, u otros efectos que si bien influyen sobre la expresión temprana de caracteres cuantitativos pueden tender a diluirse, y por lo tanto restar eficiencia a la selección.

La comparación entre distintas entidades genèticas a partir de modelos de crecimiento, que describan al proceso que conlleva a la expresión final de un determinado carácter cuantitativo, podria aportar mayores beneficios para la selección, permitiendo diferenciar qué parámetros determinantes de un proceso tienen mayores efectos en la expresión diferencial de un carácter. Metodológicamente, un enfoque que utilice criterios de información (e.g. Akaike Information Criterion) como herramienta de decisión, permitiria mayor flexibilidad en las comparaciones entre tratamientos que los tests de significancia usualmente utilizados (Dayton, 2003).

\section{OBJETIVOS}

Establecer qué grado de importancia tienen los factores origen, progenie e individuo, para el tipo forestal marginal estepario, en la variación fenotipica para la altura inicial.

Determinar qué parámetros del crecimiento determinan la expresión final de la altura al segundo año en la etapa de vivero.

\section{MATERIALES Y MÉTODOS}

Se muestreó 10 poblaciones naturales elegidas al azar a lo largo del área de distribución latitudinal del tipo forestal marginal estepario (Cuadro $\mathrm{N}^{\circ} 1$ ). Dentro de estas poblaciones, se seleccionaron también al azar, individuos femeninos separados por al menos 30 metros, a fin de disminuir las probabilidades de parentesco entre las progenies. Las semillas cosechadas de dichas plantas madre fueron estratificadas durante 60 dias en arena húmeda y cámara de frio para homogeneizar la germinación. En la primavera de 2003 se estableció un ensayo de vivero en Bariloche, Argentina ( $41^{\circ} 07^{\prime} \mathrm{S}, 71^{\circ} 15^{\prime} \mathrm{W}$ ). La siembra se efectuó en contenedores forestales con sustrato inerte de arena volcánica y turba en partes iguales. Se utilizó un invernáculo de $72 \mathrm{~m}^{2}$ de superficie con control automatizado del ambiente, y se aplicó un régimen de fertiirrigación por micro-aspersión ajustado para la especie, durante dos temporadas de crecimiento. Un total de 158 familias de medios-hermanos se incluyeron en este análisis. Veintiuna plantas por progenie fueron distribuidas en un diseño de bloques aleatorizados, con tres repeticiones por tratamiento $(\mathrm{N}=3318)$. Se efectuaron nueve mediciones de la altura, medida como la distancia desde la inserción de los cotiledones hasta la yema terminal del eje principal, durante la segunda temporada de crecimiento, a los $0,75,90,103,117,133,146,170$ y 203 dias a partir del cese de la actividad de la primera temporada (fecha elegida arbitrariamente durante el periodo de reposo, en agosto de 2004). La longitud del hipocótilo se estimó mediante una muestra al azar de 200 individuos. 
Se analizó la varianza para las alturas inicial y final de la segunda temporada de crecimiento, mediante un modelo anidado de efectos mixtos, en el cual los origenes y las progenies se utilizaron como factores aleatorios; los componentes de la varianza se calcularon para ambos factores mediante el método REML del PROC VARCOMP (SAS Institute, 2001). El modelo empleado fue:

$$
y_{i j k}=\mu+o_{i}+p_{j}\left(o_{i}\right)+b_{k}+\varepsilon_{i j k}
$$

donde $y_{i k}=$ ijkésima observación; $o_{i}=$ efecto del iésimo origen; $p_{j}=$ efecto de la jésima progenie (anidada en origen); $b_{k}=$ efecto del késimo bloque (considerado como efecto fijo) y $\varepsilon_{i j k}=$ error.

Se efectuaron análisis de correlación $(\alpha=0,01)$ para cada familia, a fin de determinar el grado de asociación entre las alturas individuales dentro de familias al inicio y al final de la temporada de crecimiento analizada. El mismo análisis se efectuó dentro de cada origen, para establecer la asociación para las alturas inicial y final promedio de las progenies.

Con el objetivo de describir al crecimiento durante la segunda temporada, se ajustaron y compararon diferentes modelos de regresión lineal y no lineal mediante el programa Prism4 (GraphPad, 2004), a nivel de progenies (se presentan en este trabajo los resultados de los mejores modelos obtenidos). La variable respuesta (altura en $\mathrm{mm}$ ) fue transformada mediante $\log _{10}$ para corregir la heterogeneidad de varianzas (Zar, 1999). Los modelos de crecimiento ajustados, y sus parámetros, fueron comparados mediante el Criterio de Información de Akaike (AIC) para 18 progenies de la población $\mathrm{M}$ (Cuadro $\mathrm{N}^{\circ} 1$ ), mediante el programa Prism4 (Motulsky \& Christopoulos, 2003). Razones de evidencia (ER: evidence ratio) mayores a 1,86 ( $65 \%$ vs. $35 \%$ de probabilidades de ser correctos dos modelos alternativos) fueron tomadas como criterio de decisión entre modelos alternativos.

Cuadro $\mathrm{N}^{\circ} 1$

POBLACIONES MARGINALES ESTEPARIAS INCLUIDAS EN EL ENSAYO DE VIVERO

\begin{tabular}{|c|c|c|c|c|c|}
\hline \multicolumn{2}{|c|}{ Población } & \multirow{2}{*}{$\begin{array}{c}\text { Latitud S } \\
37^{\circ} 08^{\prime}\end{array}$} & \multirow{2}{*}{$\frac{\text { Longitud W }}{70^{\circ} 36^{\prime}}$} & \multirow{2}{*}{$\frac{\text { Altitud (msnm) }}{1.450}$} & \multirow{2}{*}{$\frac{N^{0} \text { de progenies ensayadas }}{11}$} \\
\hline Mo & Cañada Molina & & & & \\
\hline $\mathbf{R a}$ & Cañada Rahueco & $37^{\circ} 10^{\prime}$ & $70^{\circ} 36^{\prime}$ & 1.500 & 11 \\
\hline $\mathbf{R i}$ & Riscos Bayos & $37^{\circ} 59^{\prime}$ & $70^{\circ} 47^{\prime}$ & 1.350 & 6 \\
\hline N & Catán-Lil & $39^{\circ} 21^{\prime}$ & $70^{\circ} 39^{\prime}$ & 1.100 & 11 \\
\hline$Y$ & Chacay & $40^{\circ} 51^{\prime}$ & $70^{\circ} 59^{\prime}$ & 1.250 & 14 \\
\hline $\mathrm{CH}$ & Chacabuco & $40^{\circ} 39^{\prime}$ & $71^{\circ} 01^{\prime}$ & 900 & 24 \\
\hline $\mathbf{P}$ & Pilcaniyeu Norte & $41^{\circ} 13^{\prime}$ & $70^{\circ} 42^{\prime}$ & 1.100 & 19 \\
\hline PD & Pilcaniyeu Sur & $41^{\circ} 14^{\prime}$ & $70^{\circ} 42^{\prime}$ & 1.100 & 14 \\
\hline M & El Maitén & $42^{\circ} 02^{\prime}$ & $71^{\circ} 12^{\prime}$ & 750 & 25 \\
\hline Q & Leleque & $42^{\circ} 20^{\prime}$ & $71^{\circ} 09^{\prime}$ & 850 & 23 \\
\hline
\end{tabular}




\section{RESULTADOS}

La altura promedio general al inicio de la segunda temporada de crecimiento ( $h 0$ ) fue de $64 \pm 16 \mathrm{~mm}$ (la longitud promedio del hipocótilo fue de 19,3 $\pm 4,9 \mathrm{~mm}$ ). La altura al final del período ( $h f$ ) tuvo un promedio de $216 \pm 65 \mathrm{~mm}$, con un minimo y un máximo absolutos de 65 y $512 \mathrm{~mm}$, respectivamente.

A nivel de progenies, las alturas iniciales promedio variaron entre 42 y $92 \mathrm{~mm}$, y las finales entre 145 y $320 \mathrm{~mm}$. Los valores de alturas iniciales ( $\mathrm{hOmp}$ ) y finales ( $\mathrm{hfmp}$ ) promedio para las familias estuvieron significativamente correlacionados $(r=0,55 ; p<0,01)$ (Figura $\mathrm{N}^{\circ} 1$ ). A nivel de individuos dentro de familias, ho y $h f$ estuvieron correlacionadas significativamente sólo en el $55 \%$ de las progenies $(p<0,01)$, con coeficientes de correlación mayores que 0,63 . En la Figura $\mathrm{N}^{\circ} 2$ se muestran ejemplos de los diferentes grados de asociación entre las alturas iniciales y finales de las plantas dentro de familias.

Para $h 0$ aproximadamente un $3,4 \%$ de la variación observada fue atribuible a diferencias entre origenes $(p<0,001)$, mientras que las progenies explicaron alrededor del $22,5 \%$ del total $(p<0,001)$. El $74,1 \%$ restante (residual) se debió principalmente a diferencias entre los individuos dentro de las familias. Para $h f$ los porcentajes fueron similares: 2,9 \% (origen), 24,6 $\%$ (progenie) y $72,5 \%$ (residual).

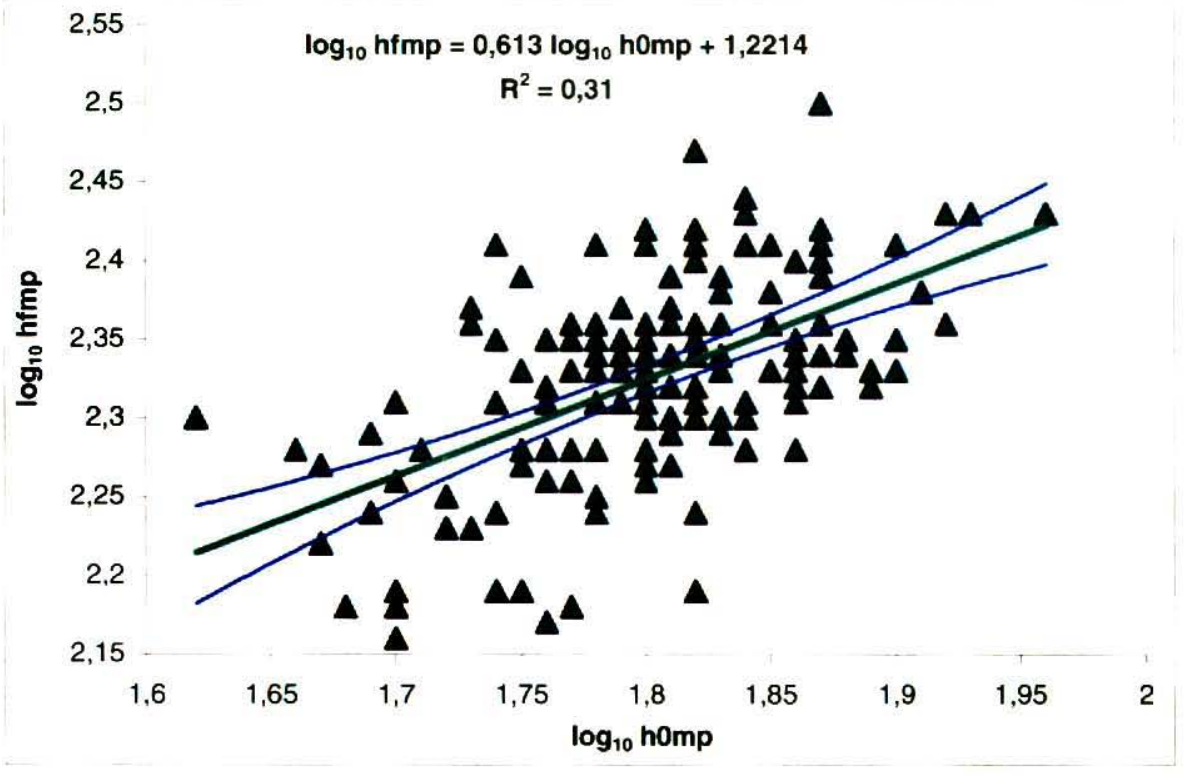

Figura $\mathrm{N}^{\circ} 1$

ASOCIACIÓN ENTRE LAS ALTURAS INICIAL Y FINAL PROMEDIO PARA LAS 158 FAMILIAS ANALIZADAS

(Lineas en azul: intervalo de confianza) 


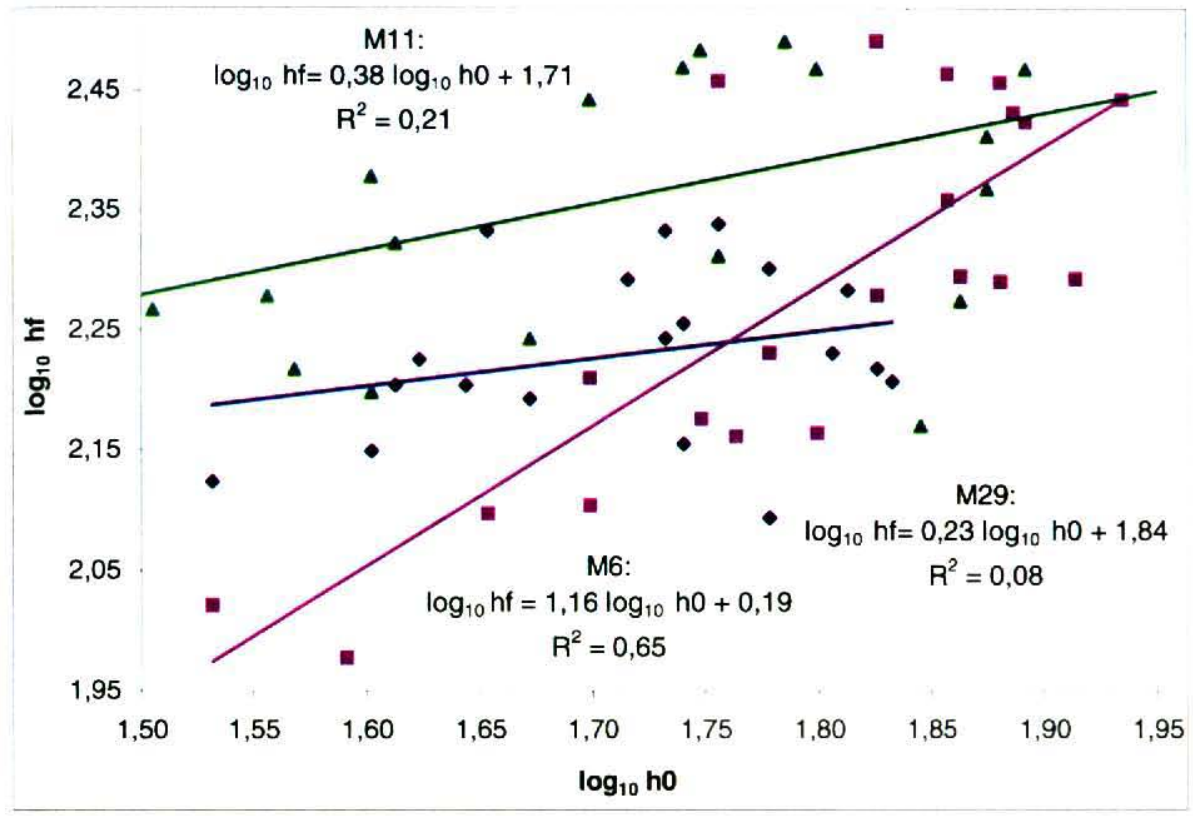

Figura $\mathrm{N}^{\circ} 2$

DIFERENTES GRADOS DE ASOCIACIÓN ENTRE LAS ALTURAS INICIALES Y FINALES DE LAS PLANTAS DENTRO DE FAMILIAS

M29 (no significativa); M11 $(r=0,45 ; p=0,04) ; \operatorname{M6}(r=0,81 ; p=0,00001)$

Los modelos que produjeron los mejores ajustes y que de manera más simple describieron al crecimiento en altura durante la segunda temporada en los distintos grupos analizados, fueron los polinomiales de segundo grado, de la forma:

$$
y^{\prime}(t)=c+b \cdot t+a \cdot t^{2}
$$

donde $y^{\prime}$ es el $\log _{10}$ de la variable respuesta y (altura en $\mathrm{mm}$ ), y $t$ el tiempo (en dias) transcurrido desde la medición inicial durante el periodo de reposo. Los parámetros $a$ y $b$ describen a la tasa de crecimiento, y $c$ al tamaño inicial de las plantas en el período analizado, respectivamente.

En el Cuadro $\mathrm{N}^{\circ} 2$ se muestran los parámetros ajustados para 18 familias de la población M, y la pendiente (indice de la tasa de crecimiento) en un punto cualquiera (e.g. a los 100 dias, la mitad del periodo analizado $=T C_{100}$ ) de cada curva. En el Gráfico 3 se observan las curvas ajustadas para dichas familias. El análisis de las asociaciones entre los parámetros de los modelos de crecimiento ajustados y las alturas medias al inicio y al final del periodo, mostró que las alturas finales medias se correlacionaron significativamente con la $T C_{100}(r=0,82 ; p$ $\left.=3,50 \cdot 10^{-5}\right)$, siendo el parámetro $a$ de las curvas el que mostró una asociación más alta con las hfmp $(r=0,61 ; p=0,01)$. Contrariamente, las homp no estuvieron correlacionadas ni con el 
indice de la tasa de crecimiento, ni con sus parámetros de manera individual.

\section{PARÁMETROS DE LOS MODELOS POLINOMIALES AJUSTADOS PARA 18 FAMILIAS DE LA POBLACIÓN M}

( $T C_{100}$ : pendiente de las curvas de crecimiento a los 100 dias)

\begin{tabular}{|c|c|c|c|c|c|c|}
\hline Progenie & ho (mm) & $h f(\mathrm{~mm})$ & $c$ & $b$ & a & $T C_{100}$ \\
\hline M1 & 74 & 307 & 1,845 & 0,004800 & $-8,36 \mathrm{E}-06$ & 0,003129 \\
\hline M13 & 68 & 296 & 1,815 & 0.003869 & $-3,36 \mathrm{E}-06$ & 0,003197 \\
\hline M26 & 67 & 265 & 1,803 & 0,004644 & $-7,80 \mathrm{E}-06$ & 0,003084 \\
\hline MA1 & 67 & 260 & 1,812 & 0,004301 & $-6,64 \mathrm{E}-06$ & 0,002973 \\
\hline M10 & 69 & 258 & 1,826 & 0,004166 & $-6,83 E-06$ & 0.002800 \\
\hline M11 & 56 & 239 & 1,722 & 0,004600 & $-7,15 E-06$ & 0.003171 \\
\hline M28 & 67 & 233 & 1,817 & 0,003932 & $-6,25 \mathrm{E}-06$ & 0,002682 \\
\hline M20 & 65 & 232 & 1,798 & 0,004315 & $-7,66 \mathrm{E}-06$ & 0,002783 \\
\hline M11T1 & 64 & 232 & 1,794 & 0,004428 & $-8,14 \mathrm{E}-06$ & 0,002800 \\
\hline M11T2 & 65 & 226 & 1,790 & 0,004278 & $-8,02 E-06$ & 0,002674 \\
\hline M19 & 66 & 222 & 1,804 & 0,004244 & $-8,39 E-06$ & 0.002565 \\
\hline M32 & 71 & 221 & 1,832 & 0,003830 & $-6,75 E-06$ & 0,002481 \\
\hline M7 & 55 & 220 & 1,722 & 0,004779 & $-8,99 E-06$ & 0,002981 \\
\hline M27 & 64 & 215 & 1,792 & 0,004650 & $-9,81 E-06$ & 0,002688 \\
\hline M9 & 62 & 202 & 1,769 & 0,004239 & $-8,64 \mathrm{E}-06$ & 0,002512 \\
\hline M6 & 64 & 201 & 1,789 & 0,004195 & $-8,89 E-06$ & 0,002417 \\
\hline M16 & 64 & 186 & 1,793 & 0,003925 & $-8,09 E-06$ & 0,002307 \\
\hline M29 & 53 & 172 & 1,714 & 0,004470 & $-9,61 E-06$ & 0,002549 \\
\hline
\end{tabular}




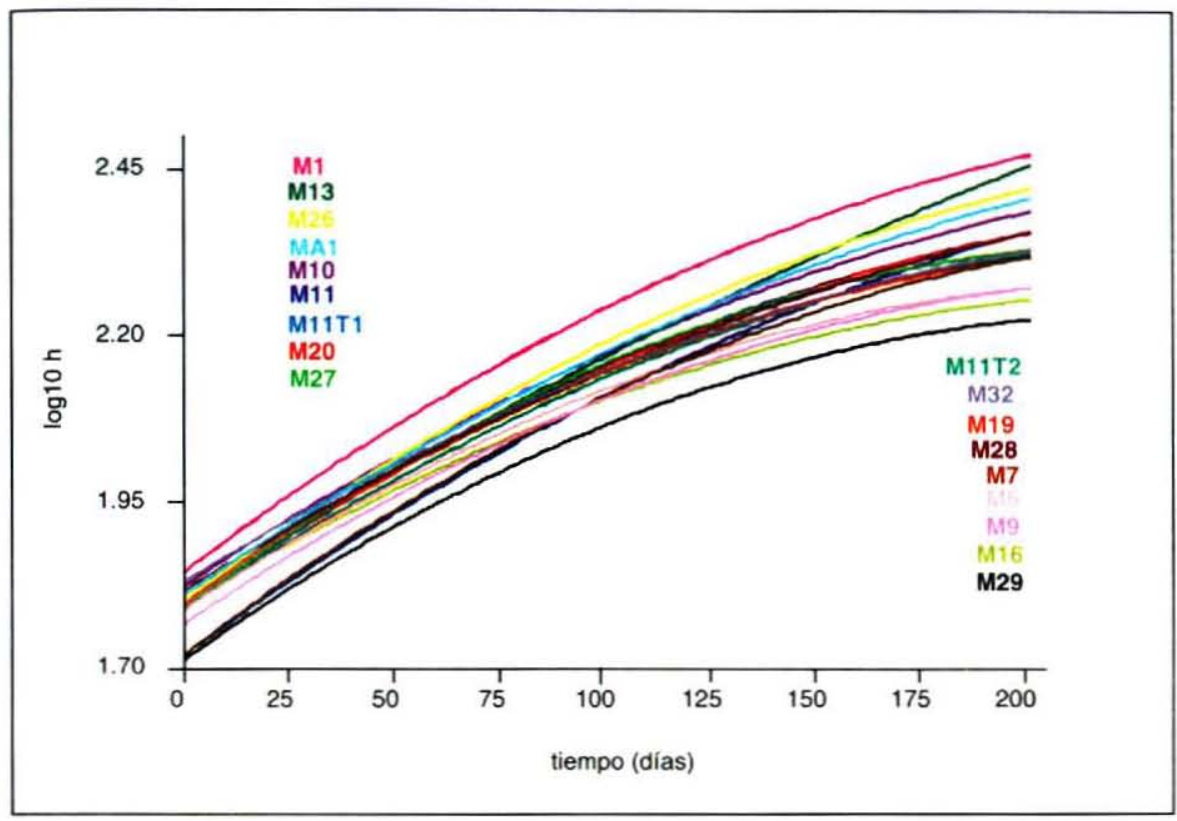

Figura $\mathrm{N}^{\circ} 3$

\section{CURVAS DE CRECIMIENTO PARA 18 FAMILIAS DE LA POBLACIÓN “EL MAITÉN"}

La comparación de modelos globales (i.e. modelo construido con el pool de datos de las 18 familias de $\mathrm{M}$ ajustándose a una sola curva), con las ordenadas al origen (parámetro c), con las pendientes (parámetros $b+a$ ), y con todos sus parámetros compartidos, versus sus respectivos modelos alternativos (curvas individuales), mostró que estos últimos tuvieron muy altas probabilidades de ser correctos (Cuadro $\mathrm{N}^{\circ} 3$ ), es decir que los sets de datos de las 18 familias no se ajustaron a una sola curva, ni compartieron sus ordenadas al origen, ni sus pendientes.

Para determinar a qué parámetros se debieron las diferencias entre las curvas de crecimiento de las diferentes familias, se compararon modelos alternativos, para cada par de familias y para cada parámetro. Es decir que para cada par de familias se efectuaron cuatro comparaciones, cada una con dos modelos alternativos. Una primera comparación global fue efectuada para establecer si los datos de dos familias se ajustaron a una misma curva ó no. Cuando esa primera comparación mostró que el modelo alternativo fue el correcto (curvas individuales mejor que una curva en común), se efectuaron tres comparaciones de modelos, con cada uno de los parámetros de la curva polinomial compartidos entre los dos sets de datos, contra los correspondientes modelos alternativos, con las dos curvas individualmente, sin el parámetro en común. En el $80 \%$ de las comparaciones efectuadas de a pares entre familias, los modelos de crecimiento ajustados difirieron globalmente o en alguno de sus parámetros. El $27 \%$ de dichas comparaciones mostró diferencias debidas al efecto de la altura inicial (parámetro $c$ ), mientras que en el $25 \%$ de los contrastes hubo diferencias en uno o en ambos parámetros de la pendiente. Sólo en el $7 \%$ de los contrastes se observaron diferencias tanto 
en la ordenada al origen como en la pendiente de las curvas. Asimismo se observó que un 22 $\%$ de las comparaciones, si bien tuvo diferencias globales, dichas diferencias no pudieron ser atribuidas a ninguno de los parámetros de los modelos en particular. En la Tabla 4 se muestran los resultados de dichas comparaciones (dado que la derivada primera de la ecuación cuadrática representa la pendiente en cualquier punto de la curva, diferencias en los parámetros b ó $a$, fueron asumidas como diferencias entre las tasas de crecimiento).

\section{Cuadro $\mathrm{N}^{\circ} 3$ \\ COMPARACIONES GLOBALES ENTRE LAS CURVAS DE CRECIMIENTO AJUSTADAS PARA CADA PROGENIE DENTRO DEL ORIGEN M}

\begin{tabular}{|c|c|c|c|c|}
\hline $\begin{array}{l}\text { Modelo más simple: Probabilidad de ser } \\
\text { correcto }\end{array}$ & & $\begin{array}{l}\text { Modelo alternativo: Probabilidad } \\
\text { de ser correcto }\end{array}$ & & ER \\
\hline $\begin{array}{l}\text { Las progenies comparten la misma curva } \\
\text { (todos sus parámetros en común) }\end{array}$ & $<0,01 \%$ & $\begin{array}{l}\text { Curvas diferentes para cada } \\
\text { progenie }\end{array}$ & $>99,99 \%$ & $\star \star \star \star$ \\
\hline $\begin{array}{l}\text { Alturas iniciales en comùn (paràmetro } c \\
\text { compartido, con } a \text { y } b \text { variables) }\end{array}$ & $<0,01 \%$ & $\begin{array}{l}\text { Alturas iniciales diferentes } \\
\text { (parámetro } c \text { no compartido) }\end{array}$ & $>99,99 \%$ & $\star \star \star *$ \\
\hline $\begin{array}{l}\text { Tasa de crecimiento en comùn } \\
\text { (parametros } a+b \text { compartidos): }\end{array}$ & $<0,01 \%$ & $\begin{array}{l}\text { Tasas de crecimiento diferentes } \\
\text { (parametros } a+b \text { no } \\
\text { compartidos): }\end{array}$ & $>99,99 \%$ & $\star \star \star \star$ \\
\hline
\end{tabular}

ER: Razones de evidencia (evidence ratio): "**: ER tendientes a infinito.

\section{Cuadro $\mathrm{N}^{\circ} 4$}

\section{COMPARACIONES DE A PARES ENTRE LAS CURVAS DE CRECIMIENTO PARA 18 PROGENIES DE M}

\begin{tabular}{|c|c|c|c|c|c|c|c|c|c|c|c|c|c|c|c|c|c|}
\hline & M1 & $\mathrm{M} 10$ & M11 & $\mathrm{M} 11 \mathrm{~T} 1$ & $\mathrm{M} 11 \mathrm{~T} 2$ & $\mathrm{M} 13$ & M16 & M19 & M20 & M26 & M27 & $\mathrm{M} 28$ & M29 & M32 & M6 & M7 & $\mathrm{M} 9$ \\
\hline MA1 & + & sd & ai & - & - & tc & tc & "tc & • & sd & tc & - & "ai tc & "tc & ttc & "ai & • \\
\hline M9 & *ai & "ai & tc & - & - & tc & so & + & • & ${ }^{*} \mathrm{tc}$ & - & •ai & "ai & "ai & sd & sd & \\
\hline$M 7$ & "ai & “ai & sod & ${ }^{*} \mathrm{ai}$ & sd & "ai tc & "ai tc & "ai & "ai & "ai & ${ }^{*} \mathrm{ai}$ & "ai tc & tc & "ai tc & "ai tc & & \\
\hline M6 & ai & * & *ai & - & sd & tc & sd & sd & - & "tc & - & $\cdot$ & -ai & - & & & \\
\hline M32 & ${ }^{*}$ tc & ${ }^{*}$ & "ai & sd & sd & tc & • & sd & sd & "tc & sd & sd & "ai & & & & \\
\hline M29 & "ai & "ai & "tc & "ai & *ai & "ai tc & *ai & "ai & "ai & *ai & "ai & "aitc & & & & & \\
\hline M28 & ${ }^{*} \mathrm{tc}$ & • & "ai tc & sd & sd & "tc & * & sd & sd & ${ }^{*} t c$ & sd & & & & & & \\
\hline M27 & "ai & tc & *ai & sd & sd & "tc & "tc & sd & sd & "tc & & & & & & & \\
\hline M26 & "ai & . & "ai & - & • & "tc & tic & "tc & - & & & & & & & & \\
\hline M2O & tai & - & "ai & sd & sd & tc & - & sd & & & & & & & & & \\
\hline M19 & tc & $\cdot$ & "ai & • & sd & ttc & • & & & & & & & & & & \\
\hline M16 & "ai tc & tc & "ai tc & ${ }^{*}$ tc & • & tc & & & & & & & & & & & \\
\hline M13 & "tc & ttc & ${ }^{*} \mathrm{ai}$ & "tc & ${ }^{*} t c$ & & & & & & & & & & & & \\
\hline M11T2 & *ai & ${ }^{*}$ & "ai & sd & & & & & & & & & & & & & \\
\hline M11T1 & ${ }^{*} \mathrm{ai}$ & - & "ai & & & & & & & & & & & & & & \\
\hline M11 & $\cdot a i$ & •ai & & & & & & & & & & & & & & & \\
\hline M10 & • & & & & & & & & & & & & & & & & \\
\hline
\end{tabular}

$\mathrm{Sd}=$ sin diferencias evidentes entre modelos; * = diferencias globales entre las curvas de crecimiento; *ai = diferencias en la altura inicial; " $t c=$ diferencias en uno o ambos parámetros de la pendiente (diferencias en las tasas de crecimiento): *ai tc = diferencias en las alturas iniciales y en las tasas de crecimiento. 


\section{DISCUSIÓN}

Los resultados de esta evaluación temprana sugieren una amplia variación individual dentro de las familias como principal fuente de variación para el crecimiento en altura. Los análisis de componentes de la varianza para las alturas finales del primero y del segundo año mostraron resultados similares. El efecto del origen sobre el crecimiento inicial en altura, si bien fue significativo, tuvo una baja participación en el total de la variación, mientras que el efecto de las progenies tuvo una participación más importante. En ambas evaluaciones fue el término residual, atribuible en gran medida a variación entre los individuos dentro de una misma familia, el que explicó el mayor porcentaje del total de la variación observada. Dadas las condiciones controladas del ensayo de ambiente común, es esperable que la mayor proporción de esa variación fenotípica detectada se deba a verdaderas diferencias genéticas.

Si bien a nivel individual hubo un cierto grado de asociación entre las alturas finales de la primera y de la segunda temporadas de crecimiento, en una proporción importante de las familias dicha asociación fue de moderada a no significativa, motivo por el cual la comparación entre individuos dentro de las progenies basada en los resultados del crecimiento en altura al primer año, no seria una opción eficiente para la selección individual temprana en vivero. A nivel de progenies las alturas inicial y final promedio mostraron una asociación significativa, aunque el análisis de regresión lineal entre ambas variables $\left(r^{2}=0,31\right)$, indicaria que la selección por la altura al final de ambos periodos de crecimiento podria mostrar resultados muy disimiles. En este sentido, las comparaciones de curvas de crecimiento entre familias de la población $\mathrm{M}$, utilizando criterios de información (AIC) como herramienta de análisis, permitieron establecer que las diferencias en el resultado final al segundo año se debieron en parte a variación en el parámetro de la altura al final del primer año, pero tambièn a diferencias en las tasas de crecimiento del segundo año, las cuales no estuvieron relacionadas al tamaño alcanzado por las plantas en su primer año.

El comportamiento de las tasas de crecimiento fue disimil para grupos de familias cuyas alturas iniciales fueron similares. Progenies que por sus alturas iniciales se ubicaron entre las mejores dentro del origen $\mathrm{M}$ (e.g. M32), durante el segundo año mostraron bajas tasas de crecimiento, y como resultado, al final del periodo se ubicaron entre las de peor altura. Asimismo, progenies que estuvieron ubicadas entre las de peor crecimiento al final del primer año, tuvieron tasas de crecimiento por encima de la media, y como consecuencia sus alturas finales fueron superiores al promedio (e.g. M11).

Los resultados de este trabajo, basado exclusivamente en el estudio de poblaciones del tipo forestal marginal estepario, no deberian extenderse a la especie sin una validación previa. Asimismo, seria de gran importancia continuar el monitoreo en plantación de las poblaciones analizadas, asi como de otras poblaciones de toda la distribución de la especie.

Se puede sugerir sin embargo, que desde un punto de vista productivo y por criterios de comportamiento inicial, se debería dar preponderancia a la selección de progenies dentro de orígenes de Ciprès, como principal estrategia para el mejoramiento genético de la especie. Restringir la búsqueda de árboles semilleros a algunos origenes elegidos por otros criterios de importancia, tales como el tamaño de sus poblaciones, la sanidad de los rodales, el estatus de conservación, las facilidades operativas, etc, aseguraria la eficiencia de los programas de 
mejora. Las pruebas de progenies en ambientes comunes permitirian obtener una importante ganancia genetica. Asimismo la selección a nivel individual en dichas pruebas de progenies podria aportar grandes ganancias, asi como también material de gran relevancia para diversos estudios genéticos.

No sólo los resultados finales de las pruebas comparativas entre entidades genéticas deberian ser tenidos en cuenta, sino también los procesos por los cuáles se llegan a dichos resultados. Las tasas de crecimiento durante el segundo año tuvieron comportamientos disimiles entre grupos de progenies con similares alturas iniciales y finales, hecho que estaria indicando la existencia de efectos maternos propios del primer año, de los cuáles hubo una cierta independencia en el comportamiento posterior de las progenies analizadas. Como ejemplo, puede mencionarse que de haberse efectuado una selección de familias en función de la altura final al segundo año, familias como M11 ó M7 podrian haberse descartado, dependiendo del umbral elegido. En cambio, tomando como criterio de selección al indice de la tasa de crecimiento, puede verse que dichas familias podrian en sucesivos periodos de crecimiento superar al promedio, a pesar de que al segundo año sus alturas fueron iguales o menores que el promedio. 


\section{CONCLUSIÓN}

Los resultados de esta evaluación temprana de origenes y progenies marginales de Ciprés de la Cordillera, nos permiten sugerir que la selección de material superior desde el punto de vista productivo, podria consistir en la generación de poblaciones de base, formadas por familias de medios-hermanos, a partir de las cuáles se deberian seleccionar los mejores individuos dentro de las familias. Una etapa de selección de familias por caracteres de crecimiento inicial, podria aportar grandes ganancias y aumentar la eficiencia de los programas de mejoramiento, disminuyendo tempranamente la cantidad de entidades genéticas a ser evaluadas a mayores plazos, y asegurando en cierta medida las ganancias obtenibles en la etapa de vivero. Metodológicamente, la determinación de diferencias en las tasas de crecimiento, obtenidas mediante mediciones sucesivas, permitiendo el ajuste y la comparación de modelos, se presenta como una alternativa que permitiria mejorar la eficiencia de los análisis usuales. Los resultados obtenidos si sólo se compararan mediciones estáticas de la altura podrian ocultar tendencias que, de mantenerse en el tiempo, resultarian en mayores ganancias. Estos análisis comparativos de progenies y origenes de Ciprés de la Cordillera se encuentran en una etapa incipiente, y se complementarán con la obtención de información en sucesivas etapas. 


\section{REFERENCIAS}

Bran D., Pérez A., Barros D., Pastorino, M. y Ayesa J., 2003. Mapa de distribución del "Ciprés de la Cordillera". Fundación Vida Silvestre Argentina, Bosques y Selvas, www.vidasilvstre.org.ar

Dayton C. M., 2003. Model Comparisons Using Information Measures. Journal of Modern Applied Statistical Methods, Vol. 2, No. 2, 281-292

Gallo L. A., Pastorino M. J. \& Donoso C., 2004. Variación en Austrocedrus chilensis (D.Don) PIC. Ser. et Bizzarri (Ciprés de la Cordillera). En: Donoso C, Prémoli A, Gallo L \& Ipinza R eds. Variación Intraespecifica en Especies Arbóreas de los Bosques Templados de Chile y Argentina: 233-251. Editorial Universitaria, Santiago.

GraphPad, 2004. GraphPad Prism version 4.03 for Windows, GraphPad Software, San Diego California USA, www.graphpad.com

Loguercio G. A., Buduba C. G. \& La Manna L., 2005. Evaluación de una reforestación con ciprés de la cordillera luego de 57 años. I Reunión sobre ecologia, conservación y uso de los bosques de ciprés de la cordillera, 28 al 30 de abril de 2005, Esquel, pp 50

Motulsky H. J. \& Christopoulos A., 2003. Fitting models to biological data using linear and nonlinear regression. A practical guide to curve fitting, GraphPad Software Inc., San Diego CA, www.graphpad.com

Pastorino M. J., 2001. Genetic Variation and Reproduction System of Austrocdrus chilensis (D. Don) Florin et Boutelje, a cypress endemic to the Andean-Patagonian Forest. Tesis doctoral presentada en la Universidad de Göttingen. Cuvillier Verlag, Göttingen, 165 pp. ISBN 3-89873033-6.

SAS Institute, 2001. SAS Ver 8.1. SAS Institute, Cary, North Carolina.

Veblen T. T., Burns B. R., Kitzberger T., Lara A. \& Villalba, R., 1995. The Ecology of the Conifers of south America. In Enright, NJ and Hill, RS. Ecology of the Southern Conifers, Melbourne University Press, 120-155

Zar J. H., 1999. Biostatistical Analysis. 4th Edition. Prentice Hall, 660 pp. 